\title{
The effect of intravitreal anti-VEGF agents on peripheral wound healing in a rabbit model
}

This article was published in the following Dove Press journal:

Clinical Ophthalmology

9 January 2012

Number of times this article has been viewed

\author{
John Christoforidis' \\ Robert Ricketts' \\ Cedric Pratt' \\ Jordan Pierce' \\ Scott Bean' \\ Michael Wells' \\ Xiaoli Zhang ${ }^{2}$ \\ Krista La Perle ${ }^{3}$ \\ 'College of Medicine, ${ }^{2}$ Center for \\ Biostatistics, ${ }^{3}$ College of Veterinary \\ Medicine, The Ohio State University, \\ Columbus, $\mathrm{OH}$, USA
}

Purpose: To investigate the effect of intravitreal pegaptanib, bevacizumab, and ranibizumab on blood-vessel formation during cutaneous wound healing in a rabbit model and to compare this effect to placebo controls.

Methods: Forty New Zealand albino rabbits underwent full thickness cutaneous wounds using 6-mm dermatologic punch biopsies. The rabbits were assigned to four groups of ten, each receiving intravitreal injections of pegaptanib, bevacizumab, ranibizumab, or no injection (untreated controls). Five rabbits from each group underwent wound harvesting on day 7 and five from each group on day 14. The skin samples were stained with hematoxylin and eosin (HE), Masson's trichrome (MT), and CD34 for vascular endothelial cells. Semiquantitative evaluation of HE- and MT-stained slides was performed by one pathologist. Quantitative assessment of mean neovascularization (MNV) scores was obtained from five contiguous biopsy margin $400 \times$ fields of CD34-stained sections by four independent observers.

Results: Week $1 \mathrm{MNV}$ scores in CD-34 stained sections were: untreated controls: $11.51 \pm 4.36$; bevacizumab: $7.41 \pm 2.82(P=0.013)$; ranibizumab: $8.71 \pm 4.08(P=0.071)$; and pegaptanib: $10.15 \pm 5.59(P=0.378)$. Week $2 \mathrm{MNV}$ data were: untreated controls: $6.14 \pm 2.25$; bevacizumab: $7.25 \pm 2.75(P=0.471)$; ranibizumab: $4.53 \pm 3.12(P=0.297)$; and, pegaptanib: $6.35 \pm 3.09$ $(P=0.892)$. Interobserver variability using intraclass correlation coefficient was 0.961 .

Conclusions: At week 1, all three anti-VEGF agents had suppressed MNV scores compared to controls. Although not statistically significant, there was an inhibitory trend, particularly with bevacizumab and ranibizumab. These effects were diminished at 2 weeks, reflecting a transition between the proliferative and remodeling phases of wound healing.

Keywords: anti-VEGF, wound healing, intraocular, pegaptanib, bevacizumab, ranibizumab

\section{Introduction}

The use of agents that suppress vascular endothelial growth factor (VEGF) is currently the standard of care for the treatment of the wet form of age-related macular degeneration. The three most commonly used agents are ranibizumab, bevacizumab, and pegaptanib. Bevacizumab and ranibizumab target all VEGF isoforms while pegaptanib specifically targets the 165 isoform of VEGF. Bevacizumab and ranibizumab are currently the mainstays of treatment of the exudative form of macular degeneration while pegaptanib is often used as maintenance. In 2010, ranibizumab was approved by the US Food and Drug Administration for the treatment of macular edema secondary to central and branch retinal vein occlusion. Both ranibizumab and bevacizumab are widely used on an off-label basis for the treatment of diabetic macular edema in the US. It is estimated that there are 1.75 million patients in the US who have the wet
Correspondence: John Christoforidis Havener Eye Institute-Retina Division, The Ohio State University, 915 Olentangy River Rd, Columbus, OH 43210, USA

$\mathrm{Tel}+$ I $614293804 \mid$

Fax +16146522610

Email john.christoforidis@osumc.edu 
form of macular degeneration with approximately 200,000 new cases of wet age-related macular degeneration diagnosed annually. ${ }^{1}$ In addition, there are expected to be 16.4 million patients with central retinal vein occlusion and branch retinal vein occlusion worldwide ${ }^{2}$ with 35,000 new cases of central retinal vein occlusion diagnosed annually in the US alone. ${ }^{3}$ While approximately 900,000 diabetic patients have vision threatening retinopathy, future projections suggest that these numbers will increase with both the aging of the US population as well as the increasing prevalence of diabetes mellitus. ${ }^{4}$ Typically, patients in these treatment groups require a mean of 3-7 intravitreal injections of an anti-VEGF agent annually per affected eye.

The side effects of systemic use of bevacizumab have been closely examined and, in addition to delayed wound healing, include hypertension, ${ }^{5-8}$ proteinuria, ${ }^{5,9}$ thromboembolic events, ${ }^{5,10}$ hematuria, ${ }^{5}$ epistaxis, ${ }^{5}$ gastrointestinal hemorrhage, ${ }^{11}$ hemoptysis, ${ }^{12}$ pulmonary hemorrhage, ${ }^{12}$ gastrointestinal perforation, ${ }^{13}$ skin rash, ${ }^{14}$ and pain. ${ }^{19}$ The effects on peripheral wound healing have been particularly well-documented since these patients often undergo tumor chemoreduction prior to surgical resection or undergo venous access port placement. Associated wound-healing complications with systemic bevacizumab include infusion port wound dehiscence, ecchymoses, incisional hernias, surgical site bleeding, and infection. ${ }^{15-19}$ These complications are especially seen in patients greater than 60 years of age. ${ }^{19}$ Although there is no clear consensus, reports from the literature for the perioperative use of systemic bevacizumab recommend that it be discontinued 6-8 weeks prior to elective surgery and started or restarted 4 weeks after surgery. ${ }^{15-22}$ Despite the deleterious effects of wound healing that have been reported with the use of systemic bevacizumab, this effect has not been specifically examined in the ophthalmology literature following intravitreally administered anti-VEGF agents. The results from the Comparison of Age-Related Macular Degeneration Treatments Trials did not specifically examine wound healing complications following intravitreal ranibizumab or bevacizumab injection. ${ }^{23}$ Perhaps because the doses of intravitreal bevacizumab are approximately 150 to 500 times less than the standard intravenous dose, it is generally presumed that these agents exert little effect systemically. However, it is imperative to study any possible effects on cutaneous wound healing as a result of intravitreal anti-VEGF therapy. Patients who receive intravitreal antiVEGF therapy are at particular risk to undergo a surgical procedure during the course of their treatment. According to the National Hospital Discharge Survey, in 2005 there were
8.86 million surgical procedures performed in the US in patients over the age of $65 .{ }^{24}$ Currently, it is unclear whether or not intravitreal anti-VEGF therapy is safe in the presence of an open wound or during the perioperative period.

Previous reports of deleterious cutaneous wound healing by systemic bevacizumab have led us to examine whether or not intravitreal anti-VEGF therapy may cause a similar effect. In this study, we specifically examined the histopathologic effects of intravitreally administered ranibizumab, bevacizumab, and pegaptanib on angiogenesis of the skin during peripheral cutaneous wound healing in a normal rabbit model.

\section{Methods}

Forty male New Zealand albino rabbits (Myrtle's Rabbitry, Thompsons Station, TN) weighing 3.8-3.9 kg were used for this study. All treatments were conducted in agreement with the ARVO Statement for the Use of Animals in Ophthalmic and Vision Research. All experimental protocols were approved, and the procedures followed were in accordance with the ethical standards of the Institutional Animal Care and Use Committee at The Ohio State University. Before the procedure, each rabbit was given $0.3 \mathrm{mg} / \mathrm{kg}$ of meloxicam PO. Anesthesia was induced with $10 \mathrm{mg} / \mathrm{mL}$ acepromazine subcutaneously and maintained with isoflurane. The rabbit's dorsal skin was shaved with electric clippers and cleansed with $10 \%$ povidone-iodine and $70 \%$ alcohol swabs before manipulation. A standard 6-mm dermatologic punch biopsy (Sklar Tru Punch, Sklar Instruments, West Chester, PA) with retention of the skin core was then created on the exposed skin, $3 \mathrm{~cm}$ lateral to the cervicothoracic vertebral column over the scapula.

The rabbits were assigned into one of four treatment groups ( $n=10$ /treatment group): ranibizumab, bevacizumab, pegaptanib, and no treatment. An intravitreal injection volume of $0.05 \mathrm{~mL}$ with either $0.5 \mathrm{mg} / 0.05 \mathrm{~mL}$ ranibizumab, $1.25 \mathrm{mg} / 0.05 \mathrm{~mL}$ bevacizumab, or $0.3 \mathrm{mg} / 0.1 \mathrm{~mL}$ pegaptanib was placed $1 \mathrm{~mm}$ posterior to the limbus of the left eye. Untreated control rabbits did not receive intravitreal injections.

Skin including the biopsy site with surrounding normal skin, was excised from rabbits under anesthesia as described above 1 week and 2 weeks post-treatment $(n=5 /$ treatment group/time point). The anesthetized rabbits were then euthanized by intravenous injection of $3 \mathrm{~mL}$ of saturated potassium chloride.

Skin samples were immediately placed into $10 \%$ neutralbuffered formalin, switched to 70\% ethanol after 24-48 hours, 
routinely processed, embedded in paraffin wax, and sectioned at $5 \mu \mathrm{m}$. Serial sections were routinely stained with hematoxylin-eosin (HE) as well as Masson's trichrome (MT) for collagen. Wound healing in HE- and MT-stained slides was semiquantitatively evaluated by an experienced pathologist (KL) blinded to experimental groups, with an Olympus BX45 light microscope with attached DP25 digital camera (B\&B Microscopes Limited, Pittsburgh, PA) according to the histologic scoring system by Abramov et al. ${ }^{25}$ In brief, acute and chronic inflammation, amount and maturation of granulation tissue, collagen deposition, re-epithelialization, and neovascularization were assessed independently and assigned a score of $0-3$. According to this scheme, a score of 0 indicates none or no cells; 1 , scant; 2, moderate; and 3, abundant. For re-epithelialization a score of 0 represents none; 1, partial; 2, complete but immature or thin; and 3, complete. For neovascularization a score of 0 indicates no vessels/high power field; 1, up to 5 vessels/high power field; 2, 6-10 vessels/high power field; and 3, more than 10 vessels/high power field. Acute inflammation was defined by the presence of neutrophils, while chronic inflammation was characterized by lymphoplasmacytic and monocytic infiltrates.

Serial paraffin sections on poly-L-lysine slides were also immunohistochemically stained for CD34 to identify vascular endothelial cells using a Dako Universal Training Center autostainer (Carpinteria, CA). Sections were deparaffinized in xylene and rehydrated prior to pretreatment with Target Retrieval Solution (Dako) in a decloaking chamber. Endogenous peroxidase was inhibited by $3 \%$ hydrogen peroxide, followed by serum-free protein block, each for 10 minutes. Sections were incubated at room temperature first with anti-CD34 (C-18) antibody (1:150, catalog \#7045; Santa Cruz Biotechnology, Inc, Santa Cruz, CA), and then with biotinylated anti-goat IgG (Vector Laboratories, Burlingame, CA), each for 30 minutes. Specific binding was amplified with RTU Vectastain Elite ABC Kit (Vector Laboratories). Chromogen reaction was developed with 3-3'diaminobenzidine solution (Dako) for 5 minutes, and nuclei were counterstained with hematoxylin.

Five contiguous $400 \times$ fields at biopsy site margins of CD34-stained sections were photographed using a sixheaded Olympus BX51 light microscope with attached Altra 20 digital camera and MicroSuite software linked to a 42" Panasonic plasma television (B\&B Microscopes Limited). By convention, the wound margin on the left aspect of the microscope slide was chosen. The photomicrographs were then randomly presented to four independent observers (JC, RR, CP, and MW) and a recorder, all blinded to experimental groups, and CD34-stained endothelial cell clusters were recorded. To reduce interobserver bias, the photomicrographs were presented in random order and each observer was blinded to the counts of the other observers. Observer means for each photomicrograph and mean neovascularization (MNV) scores representing all scores for the five photomicrographs evaluated were calculated.

Statistical analysis of HE and MT semiquantitative scores was performed using Fisher's exact test. The comparisons were between placebo and other agents at 7 days and 14 days. Standard deviations were shown with all means. CD34 endothelial cell cluster counts were first averaged across slides for each rater within each subject to avoid systematic error, and then the averaged values were analyzed using a linear mixed-effects model to account for the correlation of the observations from the same animal. In this model, the fixed effects are time, treatment, and time by treatment interaction where treatment is the three therapeutic agents and untreated controls, and time is the two time points when the measurements were made. The variance of subject and the rater by subject variation were estimated using a random statement. In the model, the variance component correlation structure was used for both as the covariance structure. In addition, the same model was used to make comparisons between the three therapeutic agents. Holm's procedure was used to adjust for multiple comparisons between the four groups at each of the two time points. After Holm's adjustment, statistical significance was considered to be present with $P<0.0063$ $(0.05 / 8)$ for the most significant ones, then with $P<0.0071$ $(0.05 / 7)$ for the second significant ones, and so on.

Intraclass correlation coefficient was used to investigate inter-rater agreement by relating the between subject variabilty to the total variability in our linear mixed model. ${ }^{26}$ All analyses were performed using SAS/STAT software (version 9.2; SAS Institute Inc, Cary, NC) using the Windows XP system.

\section{Results}

All of the rabbits that underwent intravitreal injection had clearly evident cutaneous wounds at the time of harvest. None of the wounds exhibited signs of infection at the time of harvesting.

Seven parameters of wound healing were semiquantitatively assessed in HE- and MT-stained skin sections, and none were found to be statistically significant either at 1 or 2 weeks (Table 1). While semiquantitative neovascularization scores for treated animals at 1 week were the same or slightly reduced compared to untreated controls, small-diameter 
Table I Semiquantitatively scored histologic parameters of cutaneous wound healing in normal rabbits treated with intravitreal anti-VEGF agents

\begin{tabular}{|c|c|c|c|c|c|c|c|}
\hline Treatment & Al & $\mathrm{Cl}$ & GA & GM & CD & RE & NV \\
\hline \multicolumn{8}{|l|}{7 days } \\
\hline Untreated & $2.2 \pm 0.84$ & $1.6 \pm 0.55$ & $2.2 \pm 0.84$ & $1.8 \pm 1.30$ & $1.2 \pm 0.45$ & $1.4 \pm 1.52$ & $1.6 \pm 0.89$ \\
\hline \multirow[t]{2}{*}{ Bevacizumab } & $1.2 \pm 0.45$ & $1.2 \pm 0.45$ & $1.6 \pm 0.55$ & $2.4 \pm 0.55$ & $1.6 \pm 0.55$ & $2.2 \pm 0.89$ & $1.6 \pm 0.55$ \\
\hline & $P=0.292$ & $P=0.524$ & $P=0.524$ & $P=0.714$ & $P=0.524$ & $P=0.683$ & $P=0.524$ \\
\hline \multirow[t]{2}{*}{ Ranibizumab } & $1.6 \pm 0.55$ & $1.6 \pm 0.55$ & $2.2 \pm 0.45$ & $2.0 \pm 0.00$ & $1.8 \pm 0.45$ & $2.6 \pm 0.89$ & $1.40 \pm 55$ \\
\hline & $P=0.524$ & $P=1.000$ & $P=0.524$ & $P=0.048$ & $P=0.206$ & $P=0.683$ & $P=1.000$ \\
\hline \multirow[t]{2}{*}{ Pegaptanib } & $1.6 \pm 0.55$ & $1.2 \pm 0.45$ & $1.8 \pm 0.45$ & $2.0 \pm 0.71$ & $1.6 \pm 0.55$ & $2.2 \pm 1.10$ & $1.2 \pm 0.45$ \\
\hline & $P=0.524$ & $P=0.524$ & $P=0.683$ & $P=0.714$ & $P=0.524$ & $P=0.524$ & $P=1.000$ \\
\hline \multicolumn{8}{|l|}{ I 4 days } \\
\hline Untreated & $1.0 \pm 0.0$ & $1.2 \pm 0.45$ & $1.8 \pm 0.45$ & $2.4 \pm 0.55$ & $2.2 \pm 0.45$ & $3.0 \pm 0.00$ & $1.6 \pm 0.55$ \\
\hline \multirow[t]{2}{*}{ Bevacizumab } & $1.2 \pm 0.40$ & $1.4 \pm 0.55$ & $1.8 \pm 0.45$ & $3.0 \pm 0.00$ & $2.0 \pm 0.00$ & $3.0 \pm 0.00$ & $1.4 \pm 0.55$ \\
\hline & $P=1.000$ & $P=1.000$ & $P=1.000$ & $P=0.167$ & $P=1.000$ & $P=1.000$ & $P=1.000$ \\
\hline \multirow[t]{2}{*}{ Ranibizumab } & $1.4 \pm 0.55$ & $1.4 \pm 0.55$ & $2.0 \pm 0.00$ & $2.2 \pm 0.45$ & $1.8 \pm 0.45$ & $3.0 \pm 0.00$ & $1.60 \pm 0.55$ \\
\hline & $P=0.444$ & $P=1.000$ & $P=1.000$ & $P=1.000$ & $P=1.000$ & $P=1.000$ & $P=1.000$ \\
\hline \multirow[t]{2}{*}{ Pegaptanib } & $1.8 \pm 0.45$ & $1.4 \pm 0.55$ & $2.0 \pm 0.71$ & $1.8 \pm 0.84$ & $1.8 \pm 0.45$ & $2.6 \pm 0.89$ & $2.2 \pm 0.45$ \\
\hline & $P=0.048$ & $P=1.000$ & $P=1.000$ & $P=0.524$ & $P=1.000$ & $P=1.000$ & $P=0.444$ \\
\hline
\end{tabular}

Notes: $n=5 / g r o u p ;$ mean \pm standard deviation.

Abbreviations: Al, acute inflammation; $\mathrm{Cl}$, chronic inflammation; GA, amount of granulation tissue; GM, maturation of granulation tissue; CD, collagen deposition; RE, re-epithelialization; NV, neovascularization.

neovessels were difficult to differentiate from activated fibroblasts in HE- and MT-stained sections. Therefore, CD34 immunohistochemical staining was performed for further assessment of neovascularization.

Mean neovascularization scores were calculated in wound margins from CD34-stained rabbit skin sections at 1 and 2 weeks following wounding and treatment with three different
anti-VEGF agents (Figure 1). Wound margin borders in skin sections from $39 / 40$ rabbits were clearly visible by light microscopy and were scored. The margins of one specimen (bevacizumab, 1 week) were indeterminate by all four reviewers despite sectioning deeper in the paraffin block and processing of additional skin samples, and as a result were excluded from scoring.

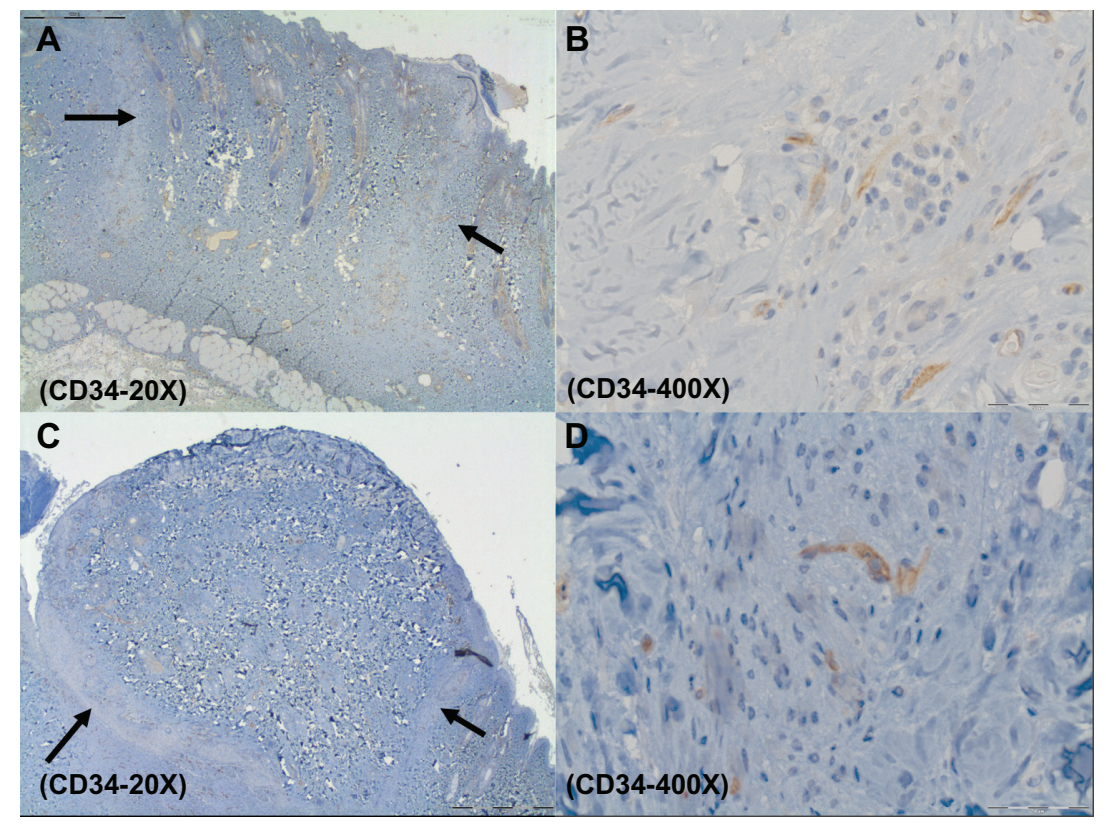

Figure I Representative CD34 histological figures of cutaneous wounds I week after wounding. At 20x wound margins are demonstrated (arrows) (A and C). At 400x, note increased endothelial cell counts in the placebo (about II) (B) versus ranibizumab (about 6) (D). 
At 1 week post-treatment, none of the treatment groups was found to inhibit, at a statistically significant level, the number of vessels compared to the placebo group. However, at this time point all of the treatment groups had lower MNV scores than untreated controls (shown as mean with standard deviation and $95 \%$ confidence interval [CI]). Compared to untreated controls (11.51 $\pm 4.36,95 \%$ CI: $9.25,13.77)$, bevacizumab (7.41 $\pm 2.82,95 \% \mathrm{CI}: 5.79,9.04, P=0.013)$ inhibited neovascularization in cutaneous wound margins. The effect was less significant for ranibizumab $(8.71 \pm 4.08$, 95\% CI: 7.00, 10.42, $P=0.071$ ); and much diminished for pegaptanib $(10.15 \pm 5.59,95 \% \mathrm{CI}: 8.16 ; 12.14, P=0.378)$. At week 2, the effect was greatly diminished as none of the agents was found to have a statistically significant inhibitory effect on neovascularization. Ranibizumab (4.53 $\pm 3.12,95 \%$ CI: $3.64,5.42, P=0.297$ ) had a lower mean MNV score compared to untreated controls $(6.14 \pm 2.25,95 \%$ CI: 4.94 , 7.34) (Figure 2). The mean MNV scores were higher than placebo for both bevacizumab $(7.25 \pm 2.75,95 \% \mathrm{CI}: 5.83$, 8.67, $P=0.471)$ and pegaptanib $(6.35 \pm 3.09,95 \% \mathrm{CI}$ : $5.11,7.59, P=0.892)$. Compared to day 7 , there was a statistically significant reduction in neovessel formation at day $14(P<0.0001)$ averaged across all treatment groups. Evaluation of interobserver variability as assessed by the intraclass correlation coefficient was 0.961 , indicating a high agreement on the reading of the slides among raters.

\section{Discussion}

In our study, all three intravitreal anti-VEGF agents were found to suppress neovascularization by qualitative assessment of MNV by CD34 immunostaining of cutaneous wound margins evaluated 1 week following treatment compared to untreated controls. Although these were not found to be statistically significant, there was an inhibitory trend, particularly with bevacizumab and ranibizumab. At 2 weeks following treatment, this effect was suppressed in all three agents, presumably because the wounds had transitioned to the remodeling phase of healing during which there are decreased numbers of blood vessels..$^{27}$ Interestingly, the bevacizumab and pegaptanib groups had higher MNV scores than the untreated group at the latter time point. Although it is not clear why this effect occurred, since the effect of anti-VEGF therapy at the maturation phase of wound healing is uncertain, this finding is not likely to be clinically significant because of the suppressed scores in all groups. Furthermore, these differences at week 2 between treatment arms and placebo were more greatly reduced than at week 1. Semiquantitative assessment of HE- and MT-stained slides did not reveal any consistent or statistically significant differences in parameters of wound healing. This may be because of the narrow scoring system applied (0 to 3 ), the small sample size per experimental group, and retention of the core at the time of punch biopsy.

This work is the first to demonstrate that intravitreally placed anti-VEGF agents can exert an effect on cutaneous wound healing by exhibiting a reduction in vessel numbers during the proliferative phase. During wound healing, angiogenesis occurs in the proliferation phase typically between days 4 and $14 .^{28}$ The new vasculature allows for cellular migration and proliferation and for the deposition of

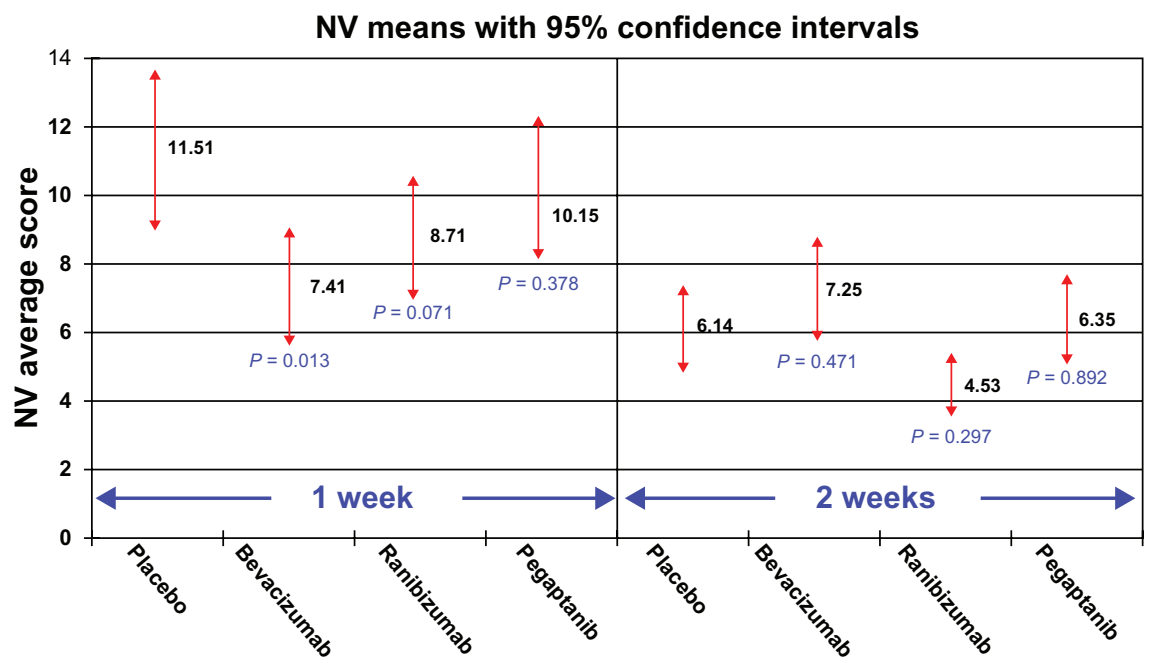

Figure 2 Mean neovascularization (MNV) scores with standard deviations and $P$-values in wound margins from CD34-stained rabbit skin sections at I and 2 weeks following wounding and intravitreal treatment with three different anti-VEGF agents. Each mean reflects endothelial cell cluster counts in $25,400 \times$ field photomicrographs/treatment group/time point. Neovascularization was significantly inhibited by all agents, most notably bevacizumab, at I week only. Note that at week 2 the differences between placebo and all three treatment arms are reduced. 
a fibrin-rich matrix that forms the granulation tissue needed to close the wound. Disturbance of angiogenesis during the proliferation phase affects the restoration of dermal integrity that is vital for re-epithelialization of a cutaneous wound. ${ }^{29}$ The proliferative phase is then followed by the maturation phase during which time there is a decrease in the number of blood vessels in the wound. ${ }^{26}$ It has been previously reported that the integrity of the wound is affected if the proliferation phase is suppressed. ${ }^{30}$ This is a likely mechanism by which systemic bevacizumab exerts its inhibitory effect on cutaneous wound healing that has been previously described in clinical studies. ${ }^{16-22}$ Further studies are warranted to evaluate whether or not intravitreal anti-VEGF therapy affects integrity and blood flow at the wound margins as well as final tensile wound strength.

There have been several clinical studies that have examined the effect of systemic bevacizumab on wound healing. In a retrospective study, Scappaticci et al ${ }^{18}$ compared wound-healing complications in patients that underwent surgery for metastatic colon cancer during treatment with and without intravenous bevacizumab. Ten of 75 bevacizumabtreated patients $(13 \%)$ compared to 1 of 29 control patients (3.4\%) had wound healing complications including bowel perforation, abdominal fistula, bowel dehiscence, hemothorax and thoracotomy wound dehiscence. While not statistically significant, these findings were found to be clinically important and the recommendation was to monitor these patients closely after surgery. ${ }^{18}$ In another retrospective study by Zawacki et al ${ }^{16}, 6 / 195$ (3.1\%) bevacizumab-treated patients compared to 0/915 controls developed wound dehiscence. Furthermore, 5/49 patients (10.2\%) receiving bevacizumab within 7 days of port placement developed wound dehiscence. These findings were statistically significant and the authors concluded that wound dehiscence after port placement was related to timing of bevacizumab therapy. They recommended delaying bevacizumab therapy for at least 28 days after major surgery. ${ }^{16}$ Two large treatment studies, Bevacizumab Regiments: Investigation of Treatment Effects and Safety (BRiTE) ${ }^{22}$ and Bevacizumab Expanded Access Trial (BEAT), ${ }^{19}$ found increased risks of wound healing complications when surgery was performed within 60 days after discontinuation of bevacizumab compared to a longer interval.

The systemic effects of intravitreal anti-VEGF agents have been investigated in several reports. Both bevacizumab and ranibizumab have been associated with low rates of hypertension and arterial thromboembolic events including cerebrovascular accident, myocardial infarction, transient ischemic attack, and angina pectoris. ${ }^{31-33}$ The safety and rates of serious adverse events of these two intravitreal agents is being further evaluated in the Comparison of Age-Related Macular Degeneration Treatments Trials Study. ${ }^{23}$ The reported incidences from these studies are small and they do not have enough statistical power to detect low rates of these events. ${ }^{34}$ These adverse events have not been associated with the use of pegaptanib, although patients with a history of myocardial infarction within 6 months and stroke within 1 year before the study were excluded from the VEGF Inhibition Study in Ocular Neovascularization (VISION) trial. ${ }^{35}$ As a result, the effects on wound healing have not been specifically addressed. In clinical practice, ophthalmologists often do not specifically ask for a patient's recent or upcoming surgical history, or examine for the presence of any cutaneous wounds at the time of intravitreal injection.

It is often assumed that intravitreal agents are minimally accessible to the peripheral circulation. Firstly, anti-VEGF agents are used in much lower quantities for treating ocular disorders than as intravenous chemotherapeutic agents. Secondly, VEGF is only one of several factors involved in angiogenesis during wound healing. ${ }^{36-38}$ Thirdly, the contents of the vitreous cavity are largely kept separate from the systemic circulation by the ocular-blood barrier. Finally, currently used anti-VEGF agents are antibodies, antibody fragments, and RNA aptamers which are large molecules by pharmacologic standards and less likely to pass through the ocular-blood barrier.

Data on the pharmacokinetics of anti-VEGF agents after intravitreal injection in humans are scarce. However, there have been several studies in animal models that have demonstrated measurable amounts of these agents in the peripheral circulation following intravitreal injection. Bilateral intravitreal injections of $0.5 \mathrm{mg}$ of ranibizumab in a nonhuman primate model showed peak serum levels of $150 \mathrm{ng} / \mathrm{mL}$ and sustained levels to greater than $10 \mathrm{ng} / \mathrm{mL}$ at 1 week. ${ }^{39}$ After intravitreal injection of bevacizumab in a cynomolgus macaque model, maximal serum levels of $1430 \pm 186 \mathrm{ng} / \mathrm{mL}$ were achieved 1 week after injection. ${ }^{40}$ Due to even smaller serum volumes, serum levels have been found to be much higher in a rabbit model $(3.3 \mu \mathrm{g} / \mathrm{mL}) 8$ days after intravitreal injection of $1.25 \mathrm{mg}$ of bevacizumab. ${ }^{41} \mathrm{With}$ regard to pegaptanib, one study demonstrated that after a single $3.0 \mathrm{mg}$ intravitreal injection, mean plasma levels were $80 \mathrm{ng} / \mathrm{mL} .^{42}$ Despite the inherent problems posed by relating human data to findings in non-neovascular animal models, it is important to realize that some amounts of these agents escape from the vitreous cavity into the serum. 
Furthermore, it would be expected that in a choroidal neovascular model there would be increased levels of the agents exiting the eye due to breakdown of the blood-eye barrier. In addition, the effect would likely be greater in humans due to the greater affinity of these agents to human VEGF.

Although the peripheral levels of anti-VEGF agents mentioned above may seem nominal, they become impressive when compared to normal serum levels of VEGF. VEGF levels in the adult human as well as the rabbit model are usually less than $100 \mathrm{pg} / \mathrm{mL}$, at least two orders of magnitude less than drug concentrations seen in the aforementioned studies. In a study by Grad et al, VEGF levels in multiple trauma patients were $742 \pm$ $152 \mathrm{pg} / \mathrm{mL}$ at their highest as compared to $82.2 \pm 10.8 \mathrm{pg} / \mathrm{mL}$ (mean \pm SEM) in healthy controls. ${ }^{43}$ In another study, normal serum and plasma levels were less than $100 \mathrm{pg} / \mathrm{mL}$ with little effect by age. ${ }^{44}$ Normal VEGF serum levels in rabbits correlate with those of humans, have been found to be less than $62 \mathrm{pg} / \mathrm{mL}$, and can increase up to $1000 \mathrm{pg} / \mathrm{mL}$ under severely hypoxic conditions. ${ }^{45}$ This amount of escape of anti-VEGF agents may be enough to suppress VEGF systemically. ${ }^{32}$

There are some inherent limitations to this study. First, the use of a rabbit model has several inherent constraints. The serum compartment is significantly smaller than that of humans, while the vitreous volume in rabbits is approximately one-third that of humans $(4.5 \mathrm{~mL}$ vs $1.5 \mathrm{~mL})$, thereby increasing systemic exposure of a humanly dosed intravitreal anti-VEGF agent. In addition, although rabbit VEGF has been found to bind to bevacizumab, ${ }^{46}$ rabbit VEGF is $94 \%$ homologous with human counterparts of VEGF 121, 165, and 189 isoforms ${ }^{47}$ An additional limitation is the exclusion of one skin sample from the 1 week bevacizumab group due to the lack of identifiable wound margins. It is uncertain how this would affect statistical significance given that the other two anti-VEGF treatment groups had similar results. Furthermore, it would be unlikely for one specimen in the bevacizumab group to significantly alter the 1-week results of our study. The controls in our study did not undergo intravitreal injection with saline or another inert vehicle. Intravitreal injection is minimally invasive and would not be expected to produce any significant elevation of serum VEGF levels. ${ }^{45}$ If such an elevation occurred, it would be expected to further increase the neovascularization in controls. Retention of the core at the time of the punch biopsy minimized the wound defect which had to heal. However, removal of the core, resulting in a larger defect to re-epithelialize and fuse would closely mimic the wounding scenario in patients and likely result in even greater anti-angiogenic effects. Lastly, we were, unfortunately, not able to directly measure ranibizumab and bevacizumab in the blood because the methodology was not available at our institution or any of our referral labs.

Although it may be presumptuous to make clinical recommendations based on the results of this preliminary study, it is important to emphasize that several reports have demonstrated the deleterious effects on wound healing by systemic bevacizumab. ${ }^{16-22}$ The amounts of these agents that escape from the vitreous into the serum have been found to be high enough to suppress peripheral VEGF. ${ }^{38-42}$ As a result, we encourage clinicians to be aware of the potential for delayed wound healing, to be observant of their patients' recent surgical history and/or the presence of any open wounds. Based on the inhibitory trend in our study during the proliferative stage of wound healing on day 7 , we recommend refraining from the use of intravitreal bevacizumab and ranibizumab in the presence of open wounds and 4-6 weeks before and 4 weeks after surgery. The use of pegaptanib or alternative forms of maintenance therapy such as photodynamic therapy could be recommended during this time interval. Finally, we suggest that patients undergoing eye surgery have any ocular wounds (ie, sclerotomy site from any gauge vitreoretinal procedures or cataract incisions) sutured when receiving intraoperative intravitreal bevacizumab. It is presumed that preoperatively administered anti-VEGF agents are washed out during the vitrectomy.

In summary, all three intravitreal anti-VEGF were found to inhibit neovascularization at cutaneous wound margins 1 week following treatment when compared to untreated controls. Although not statistically significant, there were definite inhibitory trends seen with bevacizumab and ranibizumab. Because of the large numbers of patients that may be affected by delayed wound closure after such treatments, continued investigation of the systemic effects of intravitreal anti-VEGF therapy with a larger number of subjects is warranted.

\section{Acknowledgment}

We thank Jeanne Greene and Stephanie Lewis, DVM for their veterinary support and guidance throughout the study.

\section{Disclosure}

The authors report no conflicts of interest in this work.

\section{References}

1. Friedman DS, O'Colmain BJ, Muños T, et al; for the Eye Diseases Prevalence Research Group. Prevalence of age-related macular degeneration in the United States. Arch Ophthalmol. 2004;122:564-572.

2. Rogers S, McIntosh RL, Cheung N, et al; for the International Eye Disease Consortium. The prevalence of retinal vein occlusion: pooled data from population studies from the United States, Europe, Asia, and Australia Ophthalmology. 2010;117(2):313-319. 
3. Blumenkranz MS. New therapy for central retinal vein occlusion: are intravitreal steroids a possible answer? Arch Ophthalmol. 2005;123:259-261.

4. Kempen JH, O'Colmain, Leske MC, et al; for the Eye Diseases Prevalence Research Group: The prevalence of diabetic retinopathy among adults in the United States. Arch Ophthalmol. 2004;122:552-563.

5. Yang JC, Haworth L, Sherry RM, et al. A randomized trial of bevacizumab, an anti-vascular endothelial growth factor antibody, for metastatic renal cancer. $N$ Engl J Med. 2003;359(5):427-434.

6. Pouessel D, Culine S. High frequency of intracerebral hemorrhage in metastatic renal carcinoma patients with brain metastases treated with tyrosine kinase inhibitors targeting the vascular endothelial growth factor receptor. Eur Urol. 2008;53(2):376-381.

7. Berry SR, Van Cutsem E, Kretzschmar A, et al. Final efficacy results for bevacizumab plus standard first-line chemotherapies in patients with metastatic colorectal cancer: first BEAT. J Clin Oncol. 2008; 26(Suppl 15):abstract 4025.

8. Izzedine H, Ederhy S, Goldwasser F, et al. Management of hypertension in angiogenesis inhibitor-treated patients. Ann Oncol. 2009;20:807-815.

9. Zhu X, Wu S, Dahut WL, Parikh CR. Risks of proteinuria and hypertension with bevacizumab, an antibody against vascular endothelial growth factor; systematic review and meta-analysis. Am J Kidney Dis. 2007;49(2):186-193.

10. Shah MA, Ilson D, Kelsen DP. Thromboembolic events in gastric cancer: high incidence in patients receiving irinotecan- and bevacizumabbased therapy. J Clin Oncol. 2005;23(11):2574-2576.

11. Gressett SM, Shah SR. Intricacies of bevacizumab-induced toxicities and their management. Ann Pharmacother. 2009;43(3):490-501.

12. Cho YJ, Septimiu DM, Colt HG. Bronchoscopy for bevacizumab-related hemoptysis. Lung Cancer. 2007;56:465-468.

13. Lordick F, Neinitz H, Thelsen J, Sendler A, Sarbia M. Increased risk of ischemic bowel complications during treatment with bevacizumab after pelvic irradiation: report of three cases. Int J Radiat Oncol Biol Phys. 2006;64(5):1295-1298.

14. Totlib V, Khaled S, Lapko I, Mar N, Saif MW. Skin rash secondary to bevacizumab in a patient with advanced colorectal cancer and relation to response. Anticancer Drugs. 2006;17(10):1227-1229.

15. Gordon CR, Rojavin Y, Patel M, et al. A review on bevacizumab and surgical wound healing: an important warning to all surgeons. Ann Plast Surg. 2009;62(6):707-709.

16. Zawacki WJ, Walker TG, DeVasher E, et al. Wound dehiscence or failure to heal following venous access port placement in patients receiving bevacizumab therapy. J Vasc Interv Radiol. 2009;20(5):624-627.

17. Thornton AD, Ravn P, Winslet M, Chester K. Angiogenesis inhibition with bevacizumab and the surgical management of colorectal cancer. Br J Surg. 2006;93(12):1456-1463.

18. Scappaticci FA, Fehrenbacher L, Cartwright T, et al. Surgical wound healing complications in metastatic colorectal cancer patients treated with bevacizumab. J Surg Oncol. 2005;91(3):173-180.

19. Allegra CJ, Yothers G, O'Connell MJ, et al. Initial Safety Report of NSABP C-08: A randomized phase III study of modified FOLFOX6 with or without bevacizumab for the adjuvant treatment of patients with stage II or III colon cancer (BEAT). J Clinic Oncol. 2009;27:3385-3390.

20. Bose D, Meric-Bernstam F, Hofstetter W, Reardon DA, Flaherty KT, Ellis LM. Vascular endothelial growth factor targeted therapy in the perioperative setting: implications for patient care. Lancet Oncol. 2010;11(4):373-382.

21. Ellis LM, Curley SA, Grothey A. Surgical resection after downsizing of colorectal liver metastasis in the era of bevacizumab. J Clinic Oncol. 2005;23:4853-4855.

22. Sugrue MM, Purdie DM, Feng S, et al. Serious wound healing complications (sWHC) following surgery in patients (pts) with metastatic colorectal cancer (mCRC) receiving bevacizumab (BV): results from the BRiTE observational cohort study (OCS). Proc Am Soc Clin Oncol. 2008;26(Suppl 15):abstract 4105.
23. Martin DF, Maguire MG, Ying GS, Grunwald JE, Fine SL, Jaffe GJ; for the CATT Research Group. Ranibizumab and bevacizumab for neovascular age-related macular degeneration. $N$ Engl $J$ Med. 2011;364(20):1897-1908.

24. Cullen KA, Hall MJ, Golosinskiy A. Ambulatory surgery in the United States, 2006. Natl Health Stat Report. 2009;11:1-25.

25. Abramov Y, Golden B, Sullivan M, et al. Histologic characterization of vaginal vs abdominal surgical wound healing in a rabbit model. Wound Repairs Regen. 2007;15(1):80-86.

26. Shrout PE, Fleiss JL. Intraclass correlations: Uses in assessing rater reliability. Psychol Bull. 1979;86:420-428.

27. Hunt T. Wound Healing and Wound Infection: Theory and Surgical Practice. New York: Appleton-Century-Crofts; 1980.

28. Howdieshell TR, Riegner C, Gupta V, Callaway D, Sathyanarayana, McNeil PL. Normoxic wound fluid contains high levels of vascular endothelial growth factor. Ann Surg. 1998;228:707-715.

29. Brown LF, Van De Water L, Harvery VS, Dvorak HF. Fibrinogen influx and accumulation of cross-linked fibrin in healing wounds and in tumor stroma. Am J Pathol. 1988;130:455-465.

30. Breuing K, Eriksson E, Liv P, Miller DR. Healing of partial thickness porcine skin wounds in a liquid environment. J Surg Res. 1992;52:50.

31. Howdieshell TR, Callaway D, Webb WL, et al. Antibody neutralization of vascular endothelial growth factor inhibits wound granulation tissue formation. J Surg Res. 2001;96:173-182.

32. Rich RM, Rosenfeld PJ, Pulafito CA, et al. Short-term safety and efficacy of intravitreal bevacizumab (Avastin) for neovascular age-related macular degeneration. Retina. 2006;26:495-511.

33. Michels S, Rosenfeld PJ, Puliafito CA, Marcus EN, Venkatraman AS. Systemic bevacizumab (Avastin) therapy for neovascular age-related macular degeneration twelve-week results of an uncontrolled open-label clinical study. Ophthalmology. 2005;112(6):1035-1047.

34. Csaky K, Do DV. Safety implications of vascular endothelial growth factor blockade for subjects receiving intravitreal anti-vascular endothelial growth factor therapies. Am J Ophthalmol. 2009;148(5): 647-656.

35. Chakravarthy U,Adamis AP, Cunningham ET Jr, Goldbaum M, Guyer DR. VEGF Inhibition Study in Ocular Neovascularization (V.I.S.I.O.N.) Clinical Trial Group. Year 2 efficacy results of 2 randomized controlled clinical trials of pegaptanib for neovascular age-related macular degeneration. Ophthalmology. 2006;113:1508-1521.

36. Heldin $\mathrm{CH}$, Westermark B. Mechanism of action and in-vivo role of platelet-derived growth factor. Physiol Rev. 1999;79:1283-1316.

37. Liekens S, De Clercq E, Neyts J. Angiogenesis: Regulators and clinical applications. Biochem Pharmacol. 2001;61:253-270.

38. Singer AJ, Clark RA. Cutaneous wound healing. $N$ Engl J Med. 1999;341:738-746.

39. Gaudreault J, Fei D, Rusit J, Suboc P, Shiu V. Preclinical pharmacokinetics of ranibizumab after a single intravitreal administration. Invest Ophthalmol Vis Sci. 2005;46:726-733.

40. Miyake T, Sawada O, Kakinoki M, et al. Pharmacokinetics of bevacizumab and its effect on vascular endothelial growth factor after intravitreal injection of bevacizumab in macaque eyes. Invest Ophthalmol Vis Sci. 2010;51:1606-1608.

41. Bakri SJ, Snyder MR, Reid JM, Pulido JS, Singh RJ. Pharmacokinetics of intravitreal bevacizumab (Avastin). Ophthalmology. 2007;114(5): $855-859$.

42. Macugen (pegaptanib sodium injection) product information. RX List. The Internet Drug List. Available at: http://www.rxlist.com/macugendrug.htm. Accessed February 11, 2009.

43. Grad S, Ertel W, Keel M, Infanger M, Vonderschmitt DJ, Maly FE. Strongly enhanced serum levels of vascular endothelial growth factor (VEGF) after polytrauma and burn. Clin Chem Lab Med. 1998;36(6): 379-383.

44. Larsson A, Skoldenberg E, Ericson H. Serum and plasma levels of FGF-2 and VEGF in healthy blood donors. Angiogenesis. 2002;4: $107-110$. 
45. Douzinas E, Betrosian A, Livaditi O, et al. Hypoxemic resuscitation after hemorrhagic shock is accompanied by reduced serum levels of angiopoietin-2. Cytokine. 2009;47(2):82-84.

46. Lin YS, Nguyen C, Medoza JL, et al. Preclinical pharmacokinetics, interspecies scaling and tissue distribution of a humanized monoclonal antibody against vascular endothelial growth factor. J Pharmacol Exp Ther. 1999;299:371-378.
47. Watkins RH, D’Angio CT, Ryan RM, Patel A, Maniscalco WM Differential expression of VEGF mRNA splice variants in newborn and adult hyperoxic lung injury. Am J Physiol. 1999;276(5 Pt 1):858-867.

\section{Publish your work in this journal}

Clinical Ophthalmology is an international, peer-reviewed journal covering all subspecialties within ophthalmology. Key topics include: Optometry; Visual science; Pharmacology and drug therapy in eye diseases; Basic Sciences; Primary and Secondary eye care; Patien Safety and Quality of Care Improvements. This journal is indexed on

Submit your manuscript here: http://www.dovepress.com/clinical-ophthalmology-journal

\section{Dovepress}

PubMed Central and CAS, and is the official journal of The Society of Clinical Ophthalmology (SCO). The manuscript management system is completely online and includes a very quick and fair peer-review system, which is all easy to use. Visit http://www.dovepress.com/ testimonials.php to read real quotes from published authors. 